\title{
Association of metreleptin treatment and dietary intervention with neurological outcomes in Celia's encephalopathy
}

\author{
David Araújo-Vilar $\mathbb{D}^{1,2} \cdot$ Rosario Domingo-Jiménez ${ }^{3}$ Álvaro Ruibal ${ }^{4,5,6} \cdot$ Pablo Aguiar $^{4,6}$. Salvador Ibáñez-Micó ${ }^{3}$. \\ Miguel Garrido-Pumar ${ }^{4}$ - Miguel Ángel Martínez-Olmos ${ }^{2} \cdot$ Concepción López-Soler $^{7}$ - Cristina Guillín-Amarelle ${ }^{1,2}$. \\ María González-Rodríguez ${ }^{2} \cdot$ Antonio Rodríguez-Núñez $^{8} \cdot$ Julián Álvarez-Escudero $^{9} \cdot$ Mercedes Liñares-Paz $^{10}$. \\ Blanca González-Méndez ${ }^{1}$ · Silvia Rodríguez-García ${ }^{1}$ - Sofía Sánchez-Iglesias ${ }^{1}$
}

Received: 15 May 2017 / Revised: 3 November 2017 / Accepted: 13 November 2017 / Published online: 24 January 2018

(c) European Society of Human Genetics 2018

\begin{abstract}
Celia's encephalopathy (progressive encephalopathy with/without lipodystrophy, PELD) is a recessive neurodegenerative disease that is fatal in childhood. It is caused by a c.985C $>\mathrm{T}$ variant in the BSCL2/seipin gene that results in an aberrant seipin protein. We evaluated neurological development before and during treatment with human recombinant leptin (metreleptin) plus a dietary intervention rich in polyunsaturated fatty acids (PUFA) in the only living patient. A 7 years and 10 months old girl affected by PELD was treated at age 3 years with metreleptin, adding at age 6 omega-3 fatty acid supplementation. Her mental age was evaluated using the Battelle Developmental Inventory Screening Test (BDI), and brain PET/MRI was performed before treatment and at age 5, 6.5, and 7.5 years. At age 7.5 years, the girl remains alive and leads a normal life for her mental age of 30 months, which increased by 4 months over the last 18 months according to BDI. PET images showed improved glucose uptake in the thalami, cerebellum, and brainstem. This patient showed a clear slowdown in neurological regression during leptin replacement plus a high PUFA diet. The aberrant BSCL2 transcript was overexpressed in SH-SY5Y cells and was treated with docosahexaenoic acid $(200 \mu \mathrm{M})$ plus leptin $(0.001 \mathrm{mg} / \mathrm{ml})$ for $24 \mathrm{~h}$. The relative expression of aberrant BSCL2 transcript was measured by qPCR. In vitro studies showed significant reduction (32\%) in aberrant transcript expression. This therapeutic approach should be further studied in this devastating disease.
\end{abstract}

\section{Introduction}

Electronic supplementary material The online version of this article (https://doi.org/10.1038/s41431-017-0052-8) contains supplementary material, which is available to authorized users.

$\triangle$ David Araújo-Vilar

david.araujo@usc.es

1 Thyroid and Metabolic Diseases Unit, Biomedical Research Institute (CIMUS)-IDIS, School of Medicine, Universidade de Santiago de Compostela, Santiago de Compostela, Spain

2 Division of Endocrinology and Nutrition, Hospital Clínico Universitario de Santiago de Compostela, Santiago de Compostela, Spain

3 Section of Neuropediatrics, Division of Pediatrics, Hospital Clínico Universitario Virgen de la Arrixaca-IMIB Arrixaca, Murcia, Spain

4 Division of Nuclear Medicine, Hospital Clínico Universitario de Santiago de Compostela, Santiago de Compostela, Spain
In 2013, our group described a new pediatric neurodegenerative syndrome [1]. This syndrome, termed progressive encephalopathy with/without lipodystrophy or PELD (MIM: \# 615924) is also known as Celia's encephalopathy. It has a fatal prognosis and is caused by homozygosity or

5 Fundación Tejerina, Madrid, Spain

6 Molecular Imaging and Medical Physics, Universidade de Santiago de Compostela. IDIS, Santiago de Compostela, Spain

7 Departamento de Psicología Clínica, Universidad de Murcia, Murcia, Spain

8 Pediatric Intensive Care Unit, Pediatric Area, Hospital Clínico Universitario de Santiago de Compostela, Santiago de Compostela, Spain

9 Anesthesia and Reanimation Department, Hospital Clínico Universitario de Santiago de Compostela, Santiago de Compostela, Spain

10 Department of Radiology, Hospital Clínico Universitario de Santiago de Compostela, Santiago de Compostela, Spain 
Table 1 Demographic, anthropometric, and biochemical data of the patient

\begin{tabular}{|c|c|c|c|c|c|c|c|c|c|c|}
\hline Age (years) & 3 & 3.5 & 4 & 4.5 & 5 & 5.5 & 6 & 6.5 & 7 & 7.5 \\
\hline \multirow[t]{2}{*}{ Height $(\mathrm{cm}),(\mathrm{P})$} & & 107 & 112 & 119 & 120 & - & 126 & & & 135 \\
\hline & & 98 & $(>99)$ & $(>99)$ & 98 & & 98 & & & -95 \\
\hline \multirow[t]{2}{*}{ Weight $(\mathrm{kg}),(\mathrm{P})$} & 17.5 & 19.8 & 21 & 22 & 24.2 & 25 & 25.7 & & 26.5 & 29.3 \\
\hline & 95 & 97 & 98 & 94 & 96 & 99 & 85 & & 66 & -80 \\
\hline \multirow[t]{2}{*}{$\mathrm{CP}(\mathrm{cm}),(\mathrm{P})$} & & & & 51.5 & 51.5 & & & & & 52 \\
\hline & & & & 84 & 79 & & & & & -61 \\
\hline Glucose (mg/dl) & 78 & 70 & 75 & 71 & 70 & 72 & 67 & 71 & 62 & 84 \\
\hline Insulin (mIU/l) & 163 & 12.8 & 18.9 & 17.3 & ND & 24.1 & 14.1 & 53.9 & 10.8 & 40 \\
\hline Cholesterol (mg/dl) & 144 & 160 & 165 & 145 & 146 & 128 & 124 & 123 & 157 & 197 \\
\hline Triglycerides (mg/dl) & 1150 & 286 & 166 & 199 & 237 & 104 & 58 & 186 & 98 & 196 \\
\hline LDL-c (mg/dl) & ND & ND & 101 & 76 & ND & 82 & 81 & 59 & 105 & 126 \\
\hline HDL-c (mg/dl) & 19 & ND & 31 & 29 & ND & 25 & 32 & 27 & 32 & 32 \\
\hline AST (IU/l) & 158 & 40 & 49 & 31 & 62 & 22 & 35 & 30 & 34 & 39 \\
\hline ALT (IU/L) & 304 & 59 & 68 & 35 & 98 & 41 & ND & 45 & 23 & 71 \\
\hline GGT (IU/L) & 83 & 51 & 47 & 35 & 71 & 22 & ND & 21 & ND & 39 \\
\hline Leptin (ng/ml) & 0.1 & 1.4 & 3.1 & 8.3 & ND & 2.3 & 4.5 & ND & 0.2 & ND \\
\hline DHA (mcmol/l) & ND & ND & ND & ND & ND & ND & 424 & ND & ND & 806 \\
\hline Metreleptin dose (mg qd) & 0 & 1.58 & 1.68 & 1.73 & 1.9 & 2 & 2.06 & 2.08 & 2.3 & 2.54 \\
\hline
\end{tabular}

$C P$ craneal perimeter, $P$ percentile, $N D$ not determined, $D H A$ docosahexaenoic acid

Serum leptin levels during treatment with metreleptin may be altered by leptin-binding antibodies [10] and should not be interpreted as a marker of drug effect or treatment compliance compound heterozygosity of a c.985C $>\mathrm{T}$ variant in the BSCL2 gene (NM_001122955.3). To date, seven cases have been reported $[1,2]$, with six dying before they were 9 years old (mean, 7 years; range, 6-8 years) as a consequence of the neurodegenerative process. During early infancy, psychomotor development is normal, but individuals with PELD show signs/symptoms of developmental delay around age 3 years. Subsequently, they unfailingly begin a process of rapid neurological regression and, <1 year later, they present with severe encephalopathy (Supp. material Video 1).

Studies by our group have shown that the c.985C $>$ T variant leads to aberrant splicing, causing the loss of exon 7 of the BSCL2 gene [1]. This leads to the production of an aberrant seipin protein. Seipin encoded by a transcript without exon 7 tends to form macroaggregates that cause endoplasmic reticulum stress, activating the unfolded protein response and leading to neuronal death [3].

Here we report the unexpectedly long survival of the only living patient with Celia's encephalopathy, who as of January 2017 was 7 years and 6 months old. This patient was treated with recombinant human leptin and with dietary polyunsaturated fatty acids (PUFAs) that could have some beneficial effects on neurodegeneration in terms of slowing down the process. Our in vitro studies in SH-SY5Y cells showed that leptin plus docosahexaenoic acid (DHA) significantly reduced the expression of the aberrant BSCL2 transcript, suggesting a possible mechanism of action for this treatment.

\section{Subjects and methods}

The regional IRB approved this study, which was conducted according to the ethical guidelines of the Helsinki Declaration. The patient's parents gave informed written consent for the subject's participation in the study and for the publication of clinical, imaging and genetic information.

\section{Subject}

A girl was diagnosed with PELD, which was caused by the $\mathrm{c}$. [509_513del];[985C>T] variants in the BSCL2 gene (https://da tabases.lovd.nl/shared/individuals/00002973; https://databases. lovd.nl/shared/individuals/00002973, patient ID:00002973), when she was 18 months old.

\section{Therapeutic intervention}

\section{Leptin treatment}

Due to metabolic and hepatic complications (Table 1), treatment with recombinant human leptin (metreleptin) was started when the patient was 3 years old at a dose of 0.03 
$\mathrm{mg} / \mathrm{kg}$ per day subcutaneously. The dose was titrated according to weight gain and according to the response of the metabolic and hepatic parameters (Table 1).

\section{Dietary intervention}

Our in vitro data showed that a PUFA emulsion reduced the expression of the BSCL2 aberrant transcript by around 50\% in the preadipocytes of other patient suffering from PELD [4]. In addition, supplementation with exogenous oleic acid was found to increase the motility of zebrafish that overexpressed seipin N88S [5]. Accordingly, starting when she was 6 years old, the patient began a oral diet with natural food. The diet composition was assessed with the aid of a registered nutritionist by 24-h recall questionnaires in each visit and 3-day food records. Results of nutritional calculations, using the Odimet ${ }^{\circledR}$ program (www.odimet.es) (mean \pm standard deviation) were: $1725 \pm 6.2 \mathrm{kcal}(55.1 \pm$ $1.7 \%$ carbohydrates, $17.7 \pm 1.5 \%$ proteins, and $30.0 \pm 2.0$ $\%$ fats; being saturated fat $8.77 \pm 0.72 \mathrm{~g}$ (C14:0, $0.58 \pm$ $0.11 \mathrm{~g}$; C16:0, $5.99 \pm 0.60 \mathrm{~g}$; C18:0, $1.28 \pm 0.10 \mathrm{~g}$ ), monounsaturated fat $28.93 \pm 1.30 \mathrm{~g}(\mathrm{C} 16: 1,1.14 \pm 0.03 \mathrm{~g}$; C18:1 $25.42 \pm 0.79 \mathrm{~g})$, polyunsaturated fat $14.36 \pm 1.33 \mathrm{~g}(\mathrm{C} 18: 2$ $6.62 \pm 2.70 \mathrm{~g}, \mathrm{C} 18: 33.66 \pm 1.65 \mathrm{~g}, \mathrm{C}>203.35 \pm 1.78 \mathrm{~g}$, DHA $1.52 \pm 0.22 \mathrm{~g}$, eicosapentaenoic acid (EPA) $0.85 \pm$ $0.20 \mathrm{~g}$ ), and cholesterol $230,33 \pm 65.19 \mathrm{mg}$ ). The girl also took a daily $0.57 \mathrm{~g}$ omega-3 fatty acid supplement: EpaDhax Kids 550 capsules Epadhax S.L., Boiro, A Coruña, Spain) t.i.d., each capsule contained $0.19 \mathrm{~g}$ of omega-3 fatty acids (EPA $0.09 \mathrm{~g}$, DHA $0.39 \mathrm{~g}$ ).

\section{PET and MRI studies}

Standard 18F-FDG Positron-emission tomography (PET) and volumetric magnetic resonance imaging (MRI) brainstudies were performed. PET was performed based on the European Association of Nuclear Medicine standard clinical protocols for pediatric examinations. Brain MRI was performed under anesthesia using a 1.5-Tesla magnet (Siemens Magnetom) with volumetric T1-weighted TSE, axial and coronal T2-weighted TSE, axial T1-weighted IR, T2 GRE and DWI (b values 0, 1000). PET and MRI scans were performed at the following times: just prior to the initiation of metreleptin treatment at age 3 years; at age 5 years; at age 6 years and 4 months; and at age 7 years and 6 months. All PET images were co-registered to MRI in order to combine functional and anatomical information in a common reference image. Fused PET/MRI images of the patient were obtained using a mutual information approach (Statistical Parametric Mapping, Welcome Department of Cognitive Neurology, London, UK). PET images were evaluated longitudinally by prior spatial normalization to a common reference template in order to obtain quantitative comparisons of the same anatomical region along the PET images. The metabolism in the temporal and occipital regions, thalami, caudate and putamen nucleus, cerebellum and brainstem was compared longitudinally along the PET images using the frontal cortex as a reference.

\section{Electroencephalogram (EEG)}

EEGs were recorded digitally using 21 electrodes placed according to the International 10-20 System (Nihon Kohden Europe GmbH, Rosbach, Germany).

\section{Battelle developmental inventory screening test (BDI)}

The screening version of the BDI [6] includes five domains: personal-social; adaptive; fine and gross motor; communication; and cognitive. Using these domains, the patient's developmental age was calculated when she was $24,36,53$, $64,72,77$, and 90 months old.

\section{Biochemistry}

Fasting serum samples were analyzed to determine the levels of glucose, triglycerides, total cholesterol, lowdensity lipoprotein-cholesterol (LDL-c), high-density lipoprotein-cholesterol (HDL-c), leptin and insulin, as described previously [7]. Alanine transaminase (ALT), aspartate transaminase (AST), and gamma-glutamyltransferase levels were determined with enzymatic methods on an ADVIA analyser (Siemens, Bayer Diagnostics, Tarrytown, NY, USA). DHA was measured by gas chromatography (Agilent 7890A, Supelco, Bellefonte, PA) before and during dietary intervention.

\section{In vitro studies}

\section{Plasmids}

A plasmid that encoded wild-type human seipin fused to a myc tag (6x myc-wt seipin pCS2 + MT) was the generous gift of Dr D Ito (Keio University, Japan). The Myc-fused Celia seipin expression plasmid was described previously [1]. The lentiviral plasmid containing aberrant seipin was generated as follows: aberrant seipin complementary DNA was amplified by PCR using specific primers (forward: 5'TAAGCAGGTACCTCTTTTTGCAGGATCCCATCGA-3'; reverse: 5'- TAAGCATCTAGAGCCTTGAATTCGCCCT TGAC- $3^{\prime}$ ), and the amplification product was digested with the fast-digest restriction enzymes KpnI and XbaI (cat. FD0524 and FD0684, Thermo Fisher Scientific), purified and inserted into the pLenti-CMV-GFP-2A-Puro-Blank vector 
(cat. LV073, Applied Biological Materials Inc.). The full sequence was confirmed by sequencing.

\section{Lentiviral transduction}

The SH-SY5Y neuroblastoma cell line was the kind gift of Dr Jesús Rodríguez-Requena (University of Santiago de Compostela, Spain). Cells were maintained in a 1:1 mixture of Ham's F12 (cat. N4888, Sigma-Aldrich) and Eagle's Minimum Essential Medium (MEM) (cat. M2279, SigmaAldrich) supplemented with $15 \%$ heat-inactivated fetal bovine serum (cat. 10270-106, Gibco), 1\% GlutaMax-I ${ }^{\mathrm{TM}}$ (cat. 35050-061, Gibco), 100 units $/ \mathrm{ml}$ penicillin, $100 \mu \mathrm{g} / \mathrm{ml}$ streptomycin (cat. 15140-122, Gibco), and 1\% Minimum Essential Medium non-essential amino-acid solution (cat. 11140050 , Gibco). Cells were grown in a $5 \% \mathrm{CO}_{2}$ atmosphere in a humidified incubator at $37^{\circ} \mathrm{C}$. The medium was replaced every 3 days, and the cells were sub-cultured once $90 \%$ confluence was reached. In all of the experiments, the cells were used at early passages (below P17). For lentiviral transduction, cells were seeded into six-well plates (cat. 3516 , Corning, Costar) at a density of $5 \times 10^{3}$ cells per $\mathrm{cm}^{2}$. Twenty-four hours after seeding, the medium was removed, and the cells were washed with phosphate-buffered saline (PBS). Viral particles (aberrant seipin or the empty vector as a control; functional titers of $>109$ transducing units $/ \mathrm{ml}$ produced by Cyagen Biosciences (Guangzhou, China), were added at a multiplicity of infection of 200 viral particles/cell to serum-free medium in the presence of Lentiblast A/B (1:100/1:1000, Oz Biosciences, Marseille, France). Serum was added $4 \mathrm{~h}$ after initial infection (final infection volume, $1 \mathrm{ml}$ ). Twenty-four hours after infection, the medium was removed and the cells were washed with PBS. The cells were then cultured with SH-SY5Y medium. Puromycin dihydrochloride $(2 \mu \mathrm{g} / \mathrm{ml}$ final concentration; cat. P8833, Sigma-Aldrich) was added to the cell culture medium every 2-3 days until resistant stable cells had grown. Cells were then grown routinely as described above.

\section{DHA and leptin treatment}

DHA (cat. 2534, Sigma-Aldrich) was dissolved in absolute ethanol $(100 \mathrm{mg} / \mathrm{ml})$ and then conjugated to fatty acid-free, endotoxin-free bovine serum albumin (BSA, cat. A8806, Sigma-Aldrich) at a fatty acid:BSA molar ratio of 4:1 (200 $\mu \mathrm{M}$ DHA:50 $\mu \mathrm{M}$ BSA). The solution was vortexed thoroughly for $2 \mathrm{~min}$ and incubated at $37^{\circ} \mathrm{C}$ for $2 \mathrm{~h}$ with vortex mixing every $15 \mathrm{~min}$. Stably transfected cells were seeded into six-well plates at density of $10 \times 10^{3}$ cells per $\mathrm{cm}^{2}$ and allowed to adhere for 2 days. Subsequently, the cells were incubated for $24 \mathrm{~h}$ with or without DHA $200 \mu \mathrm{M}$ conjugated to fatty acid-free BSA plus human leptin $(0.001 \mathrm{mg} / \mathrm{ml}$, cat. L4146, Sigma-Aldrich). The leptin dose was chosen based on the dose used in human treatment, assuming a distribution volume of 5 litres. Medium with $0.66 \%$ ethanol (v/v) and $50 \mu \mathrm{M}$ fatty acid-free BSA was used for untreated cells. Cells were harvested the next day. This experiment was repeated three times.

\section{RNA extraction and retrotranscription}

Total RNA was extracted from cells using Trizol (Invitrogen, Madrid, Spain) as per the manufacturer's instructions. The concentration and purity of each sample was determined by spectrophotometer (ND2000; Nanodrop). RNA samples were stored at $-80^{\circ} \mathrm{C}$ until use. The RNA was reverse transcribed using $\mathrm{M}-\mathrm{MLV}$ reverse transcriptase (Invitrogen) as described previously [8].

\section{Real-time RT-PCR}

Specific primers and probes were designed by the Universal ProbeLibrary (Roche Diagnostics, Sant Cugat del Valles, Spain) and used in a LightCycler 2.0 (Roche Diagnostics) to determine the specific expression of the BSCL2 transcript without exon 7. The reverse primer, 5'-AGCGATCATT GAGATCCACA-3', was used with probe \#42 (5'-CATC CAGC-3'); the forward primer, 5'-TTTTCGGATGTT AACCTGAGC-3', exclusively hybridized with the union of exon 6 and exon 8 (amplicons, 96 nucleotides). Real-time RT-PCR conditions are available upon request. The results were normalized to the $18 S$ gene using the $2^{-\Delta \Delta}$ CT method [9].

\section{Statistical analysis}

Real-time PCR analyses were performed in duplicate. Statistical significance was determined using a non-parametric Mann-Whitney test. Data are presented as means \pm SD with the statistical significance set at $p<0.05$. All statistical analyses were performed using SPSS for Mac (release 22.0; SPSS, Chicago, IL, USA).

\section{Results}

\section{Case report since diagnosis until the onset of metreleptin treatment}

The girl, who was 7 years and 10 months old in May 2017, appeared normal at birth. Starting when she was just a few months old (Table 2), the patient showed a generalized lipodystrophy, with well-defined musculature and hepatomegaly. From a neurological point of view, her psychomotor development was normal in terms of milestones, although with slight delays, and there was no evident 


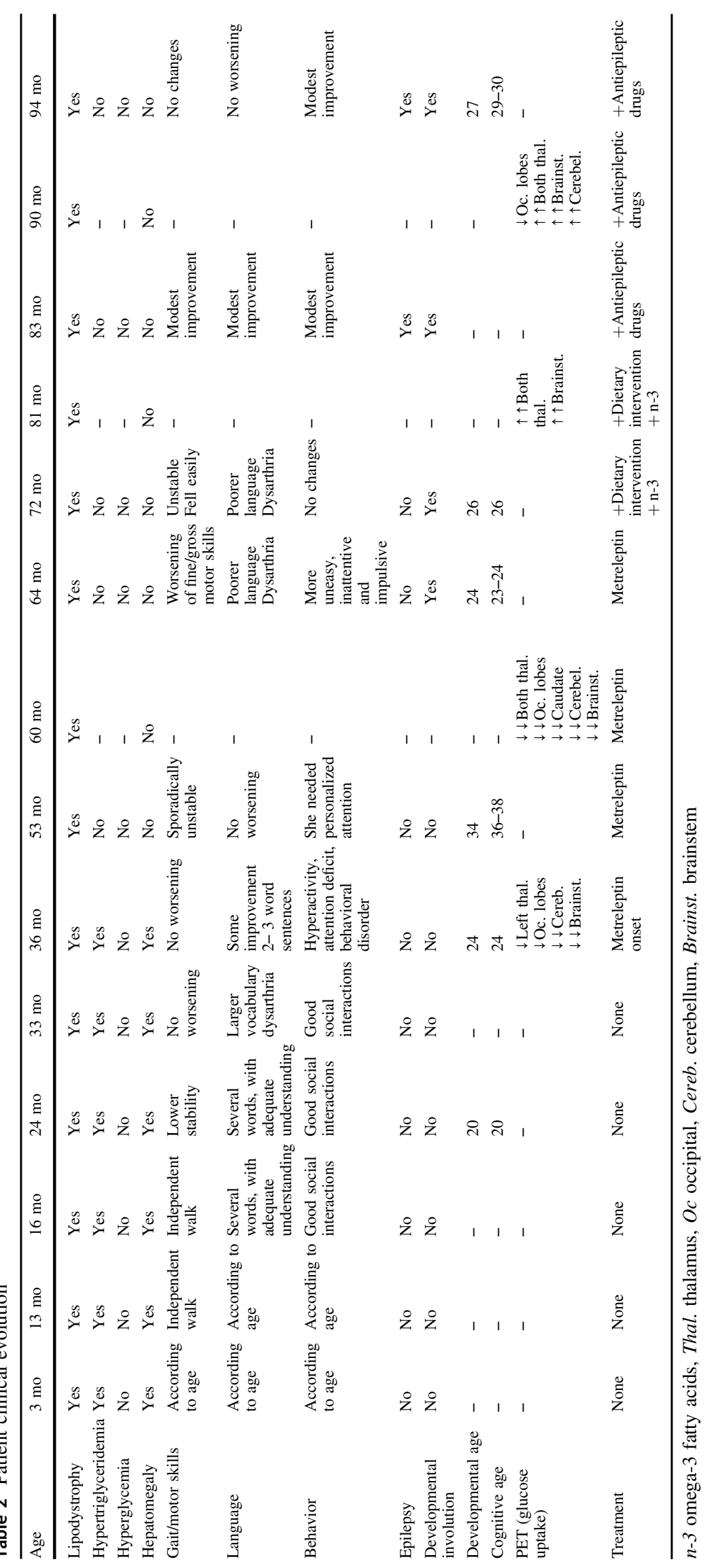


regression in the first 2 years of life. At around 24 months, her gait stability was lower than expected for her age but without abnormal movements. At 3 years, there was some improvement in her language, but she showed slow development for her age (Table 2). At that time, a PET/MRI scan was performed that showed brain hypometabolism, which was reported in a previous publication (Fig. 1a) [1]. There was marked hypometabolism of the left thalamus at the level of the basal ganglia relative to the contralateral thalamus.

\section{Clinical evolution since the onset of metreleptin treatment until 6 years of age}

At 3 years, the patient had hypertriglyceridemia, hyperinsulinemia, and hypertransaminasemia (Table 1), so
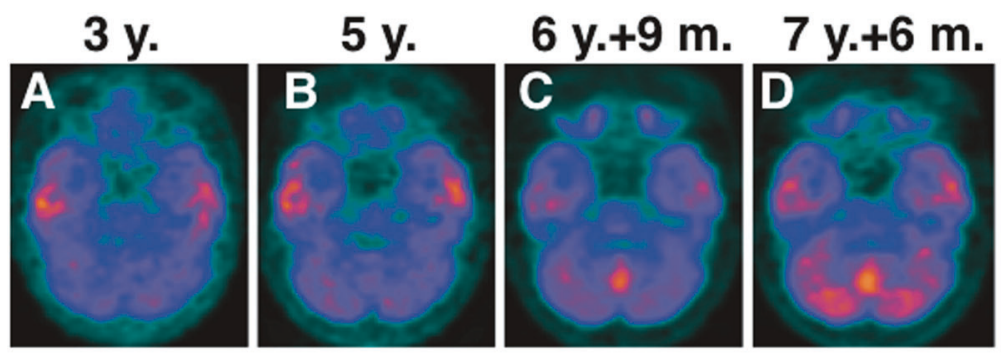

Control 1
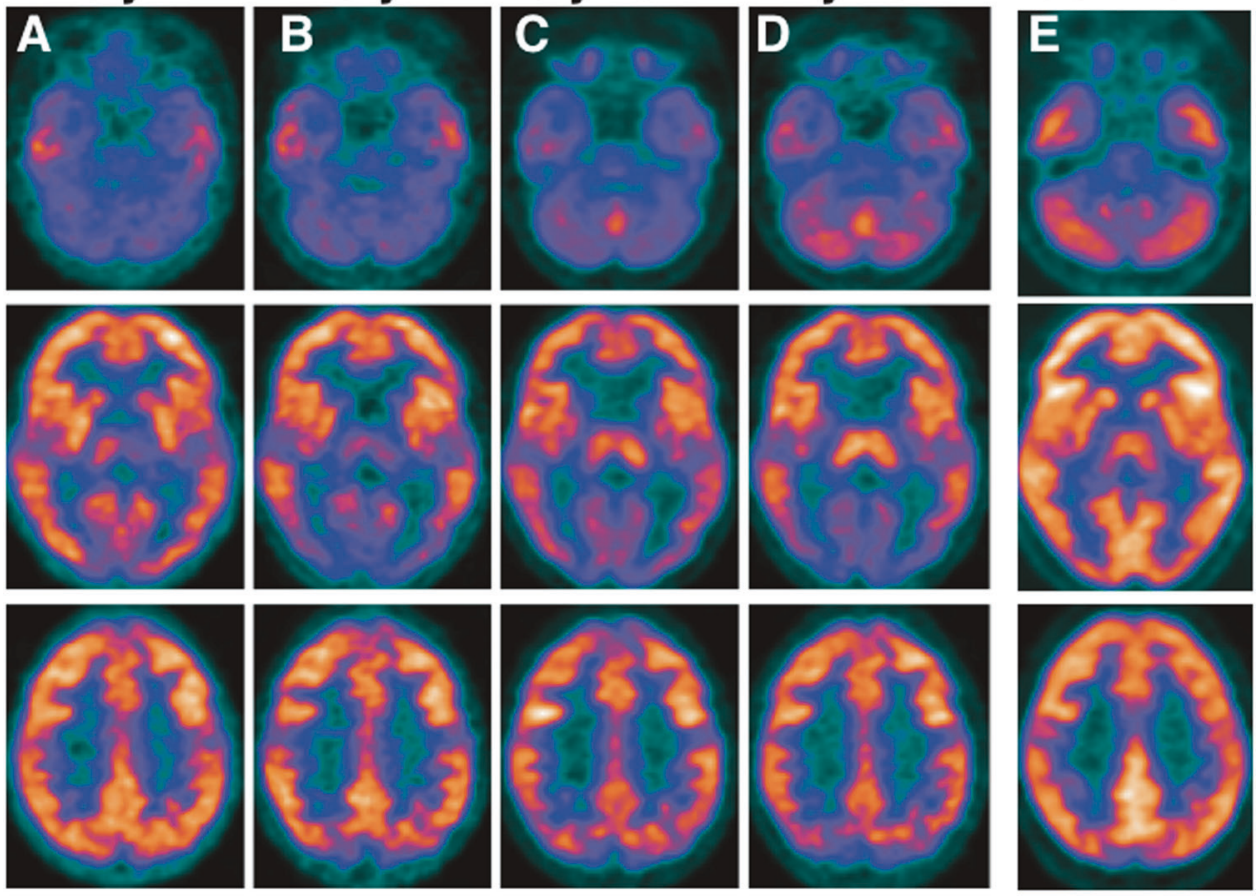

\section{Control 2}

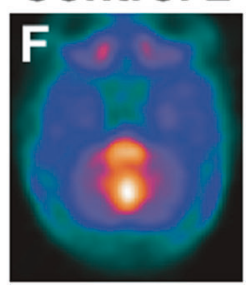

Fig. 1 Standard 18F-FDG PET brain of a girl with Celia's encephalopathy $\mathbf{a}$ at age 3 years (before therapeutical intervention); $\mathbf{b}$ at age 5 years; $\mathbf{c}$ at age 6 years and 4 months; and $\mathbf{d}$ at age 7 years and 6 months. a The images show hypometabolism at the level of both temporal lobes that is symmetrical in both hemispheres and an area of lower metabolic activity in the occipital region that is more evident in the medial planes, cerebellum, and brainstem. The remaining areas of the cerebral cortex did not show significant metabolic alterations at that time. There is marked hypometabolism of the left thalamus relative to the contralateral thalamus at the level of the basal ganglia. b The images show that metabolism in the occipital region is decreased by $-17.9 \%$. The thalamic asymmetry disappeared due to a loss of metabolic activity in the right thalamus of $-9.9 \%$, whereas the left thalamus remained unchanged $(+1.3 \%)$. There were no significant changes at the temporal lobe level, but there was a very important decrease in metabolism in both caudates nuclei $(-35.5 \%)$ plus a slight decrease in metabolism in both of the putamen nuclei $(-9.9 \%)$. Very low glucose metabolism is evident in the brainstem and cerebellar regions. c The images showed slight metabolic recovery in the occipital region $(+9.6 \%)$ and a significant increase in glucose uptake in both the thalamus $(+22.2 \%)$ and brainstem $(+25 \%)$. There were no significant changes at the temporal level, although there was a slight decrease in metabolism in both caudates $(-6.5 \%)$ and a decrease in uptake in both putamen nuclei $(-12.8 \%)$. d Compared with the images in $\mathbf{c}$, there was a reduction in glucose metabolism in the occipital region $(-14 \%)$, slight improvement in both thalami $(+6 \%)$ and temporal lobes $(+5 \%)$, no changes in the brainstem $(+2.5 \%)$ or in the caudate and putamen and clear improvement in the cerebellar region. e Standard 18F-FDG PET brain in a healthy 12-year-old girl (Control 1). f Standard 18FFDG PET brain of a newborn at age 20 days (Control 2); the image shows marked metabolism in the brainstem 


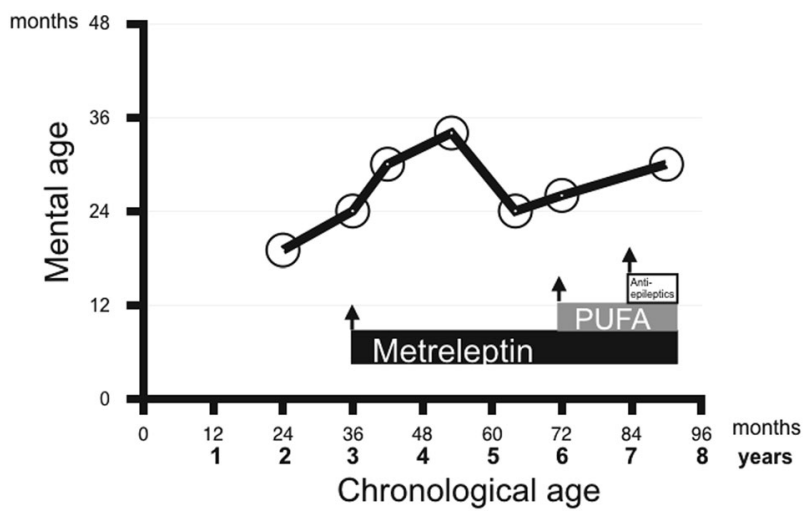

Fig. 2 The mental age of a girl with Celia's encephalopathy as calculated by the Battelle Developmental Inventory Screening Test (BDI). The BDI was performed several times along 5.5 years. The girl's mental age was always lower than her chronological age beginning when she was 2 years old. When she was 3 years old, her mental age was 2 years; this was just before she started metreleptin treatment. Her mental age progressed slowly, and she was found to have a mental age of 34 months when she was 4 years and 5 months old; subsequently, she worsened, showed a mental age of 2 years when she was 5 years and 4 months old. When she turned 6 years old, the patient began a dietary intervention with omega-3 fatty acid supplements. Her mental age started to improve and was 30 months when she was 7.5 years old

treatment with metreleptin (Aegerion Pharmaceuticals, CA, USA) was started as compassionate use. This therapy rapidly improved the patient's plasma triglyceride concentrations, insulinemia, and liver transaminase levels, and these remained acceptably reduced at the time of this report, despite plasma leptin levels were low when patient was 7 years old [10].

Although her growth was appropriate for her weight, and head circumference, which were in the normal range, as expected in Berardinelli-Seip syndrome, her height was in a higher percentile (Table 1).

In terms of neurological function, when the girl was 4 years and 11 months old, her parents reported that she was sporadically unstable. When she was 53 months old, her developmental age was only 34 months according to the BDI (Fig. 2). When she was 5 years old, a second MRI/PET scan was performed, and there was clear deterioration compared with the MRI/PET conducted when she was 3 years old. Specifically, in MRI, both caudate nuclei and thalami had decreased in size. There was hypersignal in the white matter adjacent to the body of both lateral ventricles, as well as in the region anterior to the head of both caudate nuclei. Similarly, there was a slight decrease in the volume of white matter in both outer capsules. On the other hand, PET images obtained 2 years after the onset of metreleptin treatment, when the patient was 5 years old, showed decreased metabolism in the occipital region, and the thalamic asymmetry had disappeared due to a decrease in metabolism in the right thalamus (Fig. 1b). There was a very important decrease in metabolism in both caudate nuclei.

When the girl was 5 years and 4 months old, the parents reported that they had observed significant clinical worsening in the previous few months, with regression in all areas, that is, language, cognitive and behavioral areas (Table 2). Her developmental age as calculated using the BDI was 24 months; this represented a 10-month deterioration since the previous evaluation, which was performed when she was 4 years and 5 months old.

\section{In vitro studies with SH-SY5Y cells overexpressing the BSCL2 aberrant transcript}

Our in vitro studies showed that 24-h treatment of cells with human leptin plus DHA significantly reduced the expression of the aberrant seipin transcript (an average 32\% reduction in three independent experiments) (Fig. 3).

\section{Clinical evolution since dietary intervention until 7 years and 10 months of age}

By the time the girl was 6 years old, her parents reported that she was unstable at times and fell easily (Table 2). At that point, a first EEG showed slow tracing but not the paroxysmal activity that is typical of epilepsy. Her developmental age at that time was 26 months. She was started on an oral diet that was rich in PUFAs plus a daily omega-3 fatty acid supplement. When she was 6 years and 9 months old, MRI/PET was performed; this was 2 years after the previous MRI/PET. The MRI results showed that both the

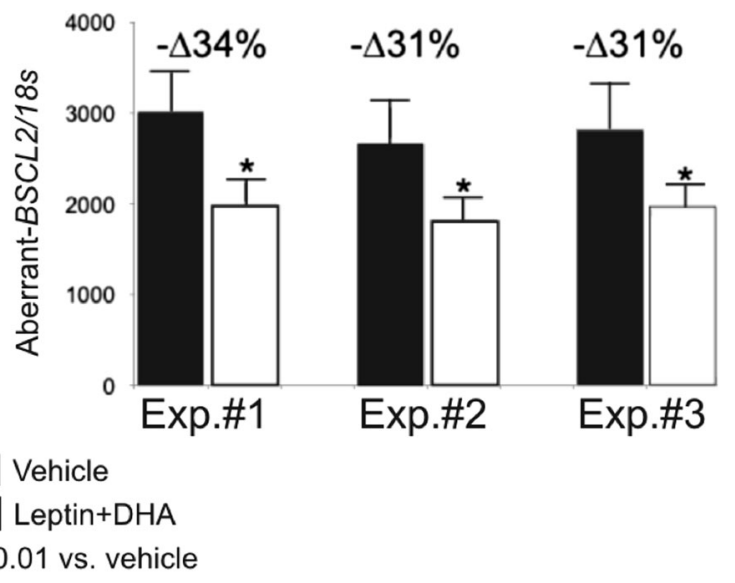

Fig. 3 The effect in vitro of docosahexaenoic acid (DHA) plus leptin treatment on SH-SY5Y neuroblastoma cells that overexpress the aberrant BSCL2 transcript. After $24 \mathrm{~h}$ of treatment, the relative expression of aberrant transcript was significantly reduced by an average of $32 \%$ in three independent experiments. Relative expression of the aberrant $B S C L 2$ transcript. ${ }^{*} p<0.01$ vs. vehicle. All samples were analyzed in duplicate, $n=3$. Results were normalized using the $18 S$ gene 
caudate nuclei and thalamus continued to decrease in size. On the other hand, a hyperintense signal was observed in the white matter adjacent to the bodies of both lateral ventricles, as well as in the region in front of the heads of both caudate nuclei. Similarly, there was a slight decrease in the volume of white matter in both of the outer capsules with respect to the 2014 study, performed when the girl was 5 years old. The PET results (Fig. 1c) showed a significant increase in glucose uptake in both thalami and in the brainstem.

The girl continued to fall, and her instability persisted. An EEG was performed when she was 6 years and 11 months old, and 31 myoclonic epileptic seizures were registered during the 3-h EEG video. Treatment with levetiracetam (until $600 \mathrm{mg}$ bid) was initiated, which reduced the number of seizures; however, valproic acid $(250 \mathrm{mg}$ b.i. d.) was added because myoclonus reappeared 2 months later. Subsequently, when the patient was 7 years and 3 months old, clonazepam ( $0.5 \mathrm{mg}$ b.i.d.) was added due to a lack of control of the myoclonic seizures, and levetiracetam and valproic acid were withdrawn.

Currently, at the age of 7 years and 10 months, the patient does not have seizures, and the neurodegenerative process appears to have improved. Compared with the previous PET scan, the most recent PET scan (at age 7 years and 6 months) showed a reduction in glucose metabolism in the occipital region; slight improvement in both thalami, temporal lobes, and brainstem; and clear improvement in the cerebellar region (Fig. 1d). Compared with the previous MRI, the most recent MRI showed a decrease in the volume of the head of both caudate nuclei and a clear loss of white matter bilaterally in the external and internal capsules. The BDI showed a developmental age of 30 months (a 4-month improvement since the previous evaluation). The final EEG showed frequent bursts of spike activity, wave activity and spike-wave activity that were synchronous and asynchronous over both central-parieto-temporo-occipital lobes (Supplementary Figure 1S). That said, the girl has a reasonably normal quality of life for her neurological age, goes to school (care for children with special needs), and interacts with her parents and younger brother. She can respond to simple commands and perform some simple tasks (Supp. material Video 2).

\section{Discussion}

Celia's encephalopathy is a devastating, extremely rare neurodegenerative disease that leads inevitably to death in childhood [1]. Starting when they are 3 or 4 years old, affected children progressively lose all neurological function, and this loss is accompanied by seizures that can barely be controlled with antiepileptic drugs.
In this study, we described the unique natural history of the only living patient (to the best of our knowledge) with Celia's encephalopathy. Currently, at age 7 years and 10 months, the girl shows obvious signs of encephalopathy, but she is not as affected as one might expect given the natural history of the other six reported cases, who died, on average, at age 7 years. If we compare the evolution of the onset of symptoms in this patient, that is, the onset of psychomotor delays, neurological regression, seizures, and death (Fig. 4), it is clear that the period of developmental delay without neurological regression lasted longer (up to 5 years) than in the other reported cases, and the neurological regression appeared to be slower. In the same way, the appearance of myoclonic epilepsy was detected much later than expected in comparison with the other six cases.

As noted, this patient started early treatment with metreleptin in order to control her metabolic disturbances. Subsequently, at 6 years of age, her diet was modified by increasing the proportion of PUFAs and adding omega-3 supplementation. Before this intervention, functional impairment was evident in the cerebral PET images, especially in the basal ganglia, the temporal and occipital lobes and the cerebellum and brainstem. Interestingly, the last two PET scans, one when the patient was 6 years and 9 months old and one when she was 7 years and 6 months old, showed improvement in previously affected areas, except for the caudate and putamen nuclei and the occipital lobes. Most notably, both thalami showed increased 18-F-glucose uptake $(+20 \%)$, and also the cerebellar regions and brainstem experienced a striking improvement.

Regarding higher function and motor skill development, the child showed gradual deterioration from age 2 years until the present, although the deterioration occurred at a considerably slower rate than in the other cases. Strikingly, she showed modest improvement over the last 18 months according to the BDI.

In light of these results, it is tempting to speculate that the combination of metreleptin plus increased dietary PUFA levels positively affected the patient by slowing the neurodegenerative process. There is a reasonable body of scientific evidence suggesting that leptin has a beneficial effect on central nervous system function $[11,12]$ and on some neurodegenerative disorders, like Alzheimer's disease (AD) [13, 14]. In addition to its well-known effects on the regulation of food intake and on energy expenditure in the hypothalamus [15], leptin can affect neurogenesis, synaptogenesis, neuronal excitability, axon growth and dendritic morphology and can have neuroprotective effects [12, 16-21]. Moreover, leptin regulates the synapse morphology of hippocampal neurons [22] and modulates the development of oligodendroglial cells [23], which may contribute to structural changes in gray matter. 
Fig. 4 The natural history of Celia's encephalopathy in the seven patients reported to date. Panel a shows the six patients who died in childhood.

Developmental delays started at 2 years of age and extended until age 3.5 years (purple).

Neurological regression was subsequently apparent (red), and seizures (orange) began before age 4 years. The patients died at an average age of 7 years (black). Panel b shows the disease time-course of the patient who is currently alive. Development delays started at 2 years of age and extended until age 5 years (purple); subsequently, neurological regression was apparent (red), and seizures appeared at 6 years and 11 months of age (orange).

Therapeutic intervention is represented by arrows: metreleptin (green), dietary intervention (blue), and antiepileptic drugs (black)

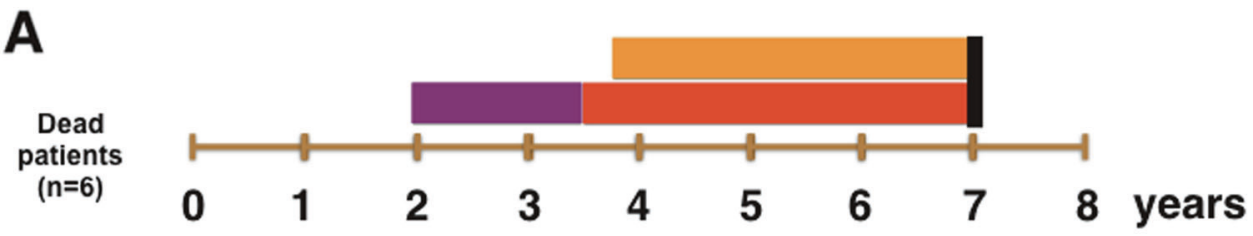

B

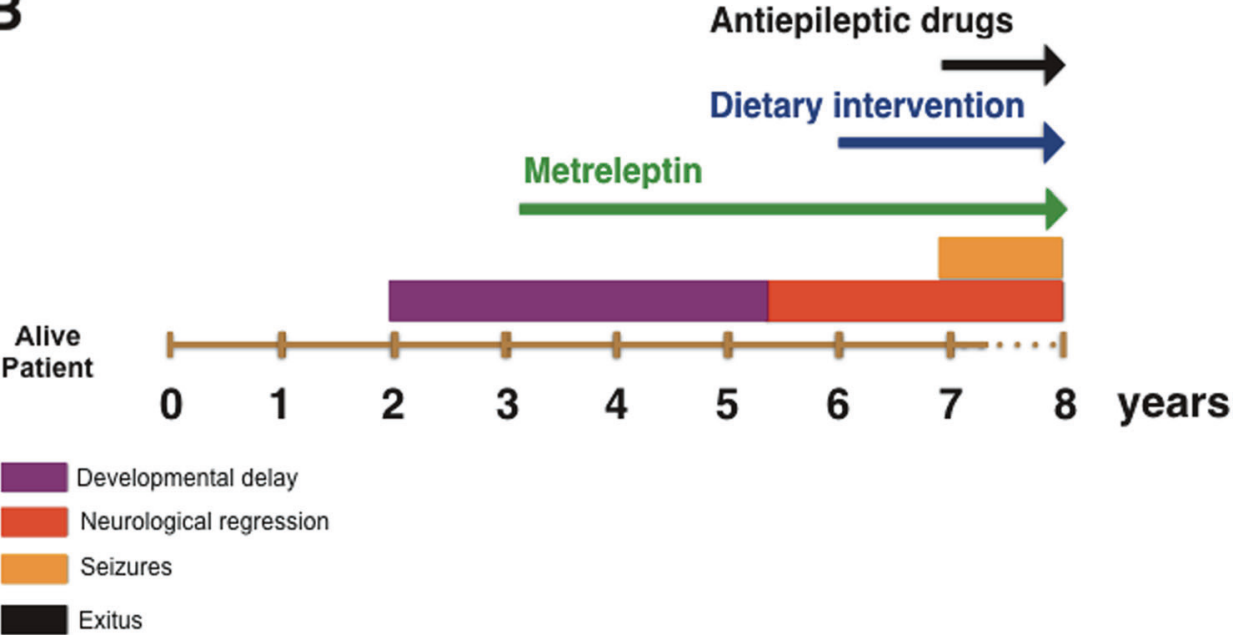

In addition, leptin may help prevent neuronal death in neurodegenerative disorders [12]. For example, in transgenic mice models of $\mathrm{AD}$, leptin replacement therapy (LRT) results in significant cognitive improvement [13] and reduces the brain amyloid-beta peptide load [14]. The neuroprotective effects of leptin involve the following: activation of the Janus Kinase 2-Signal transducer and activator of transcription 3, Protein Kinase B, and mitogenactivated protein kinases/extracellular signal-regulated kinases signaling pathways [24]; inhibition of apoptotic cell death and improved cell survival via the regulation of apoptotic enzymes; protection against glutamatergic cytotoxicity; defense against oxidative stress via expression of the membrane antioxidant MnSOD and stabilization of mitochondrial membranes; and the promotion of hippocampal progenitor cell proliferation [12]. Interestingly, high circulating leptin levels in humans are associated with a reduced incidence of dementia $[19,25]$, and leptin signaling seems to be dysregulated in the brains of AD patients [26]. Notably, in leptin deficiency disorders such as lipodystrophy and congenital leptin deficiency (CLD), LRT results in different degrees of improvement in some brain functions. For example, gray matter density is increased in diverse brain regions in CLD that is treated with leptin, and improvements in neurocognition have been reported in isolated cases [27]. In lipodystrophic patients, LRT increases food-related neural activity in the orbitofrontal cortex and suppresses activity in the amygdala, hippocampus, insula, caudate, and putamen [11].

Regarding dietary intervention, there are some studies that support the role of dietary PUFAs, particularly DHA, on brain development in children [28-31], and it may also have beneficial effects on neurodegenerative diseases such as $\mathrm{AD}$, Parkinson's disease, attention deficit hyperactivity disorder, autism, and traumatic brain injury [32-36], although some results are controversial $[37,38]$. DHA is the most abundant omega-3 long-chain PUFA in nerve cells, and it largely determines the structural and physicochemical properties of the plasma membrane, including membrane viscosity, lateral mobility, phase separation and microdomain segregation, conformational transitions and the stability of membrane proteins and lipid-protein and protein-protein interactions [39]. DHA modulates neurogenesis, synaptogenesis, and neurite outgrowth; refines synaptic connectivity; controls neurotransmitter release; plays a role in memory consolidation processes [29, 40, 41]; and is protective against trauma or ischemic insults. All of these processes are highly relevant to understanding and treating any neurological disorder. Therefore, dietary modifications and supplementation may have neuroprotective and therapeutic effects [42]. However, we caution that too much omega-6 during neural development in children negatively affects neurite growth and may contribute to poor neurodevelopment [43]. In addition, altering the omega-6:omega-3 ratio during early life may induce 
developmental changes in brain connectivity, synaptogenesis, cognition and behavior [44].

Although we are aware that this could be a case of variable expression, as happens in other Mendelian diseases, the natural history of the other six cases was remarkably consistent. Also it is possible that antiepileptic therapy could have some effect on the BDI results. However, the clear improvement in glucose uptake in several brain regions was not expected in a neurodegenerative disease.

Our in vitro results strongly suggested that the combination of human leptin plus DHA has some effect on the splicing rate of the aberrant BSCL2 transcript, at least in cells in which it is overexpressed. Although we do not know the precise molecular mechanisms underlying this finding, other authors have reported that DHA differentially modulates the expression of transcripts both in brain [39] and in other tissues [45].

In summary, our results suggest that treatment with recombinant human leptin plus a dietary intervention might slow down the fatal natural course of Celia's encephalopathy.

Acknowledgements We thank the patient and her parents for their cooperation and collaboration in this study. This work was supported by the Fundación Mutua Madrileña and by the Instituto de Salud Carlos III and the European Regional Development Fund, FEDER (PI13/00314). SR-G was awarded a Research Fellowship by the Asociación Española de Familiares y Afectados de Lipodistrofias (AELIP). We thank Bristol-Myers-Squibb, AstraZeneca, and Aegerion Pharmaceuticals for providing metreleptin, Dr Jesús R Requena for the kind gift of SH-SY5Y cells, and David Araujo-Gonzalez for technical support in Tube-Video presentation.

\section{Compliance with ethical standards}

Conflict of interest DA-V received an honorarium as an expert advisor from Aegerion Pharmaceuticals; the remaining authors declare that they have no conflict of interest.

\section{References}

1. Guillen-Navarro E, Sanchez-Iglesias S, Domingo-Jimenez R, et al. A new seipin-associated neurodegenerative syndrome. J Med Genet. 2013;50:401-9.

2. Alaei MR, Talebi S, Ghofrani M, Taghizadeh M, Keramatipour M. Whole exome sequencing reveals a BSCL2 mutation causing progressive encephalopathy with lipodystrophy (PELD) in an Iranian pediatric patient. Iran Biomed J. 2016;20:295-301.

3. Ruiz-Riquelme A, Sánchez-Iglesias S, Rábano A, et al. Larger aggregates of mutant seipin in Celia's Encephalopathy, a new protein misfolding neurodegenerative disease. Neurobiol Dis. 2015;83:44-53.

4. Sanchez-Iglesias S, Unruh-Pinheiro A, Guillin-Amarelle C, et al. Skipped BSCL2 transcript in Celia's encephalopathy (PELD): new insights on fatty acids involvement, senescence and adipogenesis. PLoS ONE. 2016;11:e0158874.
5. Holtta-Vuori M, Salo VT, Ohsaki Y, Suster ML, Ikonen E. Alleviation of seipinopathy-related ER stress by triglyceride storage. Hum Mol Genet. 2013;22:1157-66.

6. Newborg JSJ, Wnek L. Inventario de desarrollo Battelle. 1996.

7. Araujo-Vilar D, Loidi L, Dominguez F, Cabezas-Cerrato J. Phenotypic gender differences in subjects with familial partial lipodystrophy (Dunnigan variety) due to a nuclear lamin A/C R482W mutation. Horm Metab Res. 2003;35: 29-35.

8. Victoria B, Cabezas-Agricola JM, Gonzalez-Mendez B, et al. Reduced adipogenic gene expression in fibroblasts from a patient with type 2 congenital generalized lipodystrophy. Diabet Med. 2010;27:1178-87.

9. Livak KJ, Schmittgen TD. Analysis of relative gene expression data using real-time quantitative PCR and the 2(-Delta Delta C(T)) Method. Methods. 2001;25:402-8.

10. Chan JL, Koda J, Heilig JS, Cochran EK, Gorden P, Oral EA, Brown RJ. Immunogenicity associated with metreleptin treatment in patients with obesity or lipodystrophy. Clin Endocrinol (Oxf). 2016;85:137-49.

11. Aotani D, Ebihara K, Sawamoto N, et al. Functional magnetic resonance imaging analysis of food-related brain activity in patients with lipodystrophy undergoing leptin replacement therapy. J Clin Endocrinol Metab. 2012;97:3663-71.

12. Paz-Filho GJ. The effects of leptin replacement on neural plasticity. Neural Plast. 2016;2016:8528934.

13. Farr SA, Banks WA, Morley JE. Effects of leptin on memory processing. Peptides. 2006;27:1420-5.

14. Fewlass DC, Noboa K, Pi-Sunyer FX, Johnston JM, Yan SD, Tezapsidis N. Obesity-related leptin regulates Alzheimer's Abeta. FASEB J. 2004;18:1870-8.

15. Elmquist JK, Maratos-Flier E, Saper CB, Flier JS. Unraveling the central nervous system pathways underlying responses to leptin. Nat Neurosci. 1998;1:445-50.

16. Bouret SG. Neurodevelopmental actions of leptin. Brain Res. 2010;1350:2-9.

17. Harvey J. Leptin: a diverse regulator of neuronal function. J Neurochem. 2007;100:307-13.

18. Parimisetty A, Dorsemans AC, Awada R, Ravanan P, Diotel N, Lefebvre d'Hellencourt C. Secret talk between adipose tissue and central nervous system via secreted factors-an emerging frontier in the neurodegenerative research. J Neuroinflamm. 2016;13:67.

19. Paz-Filho G, Wong ML, Licinio J. The procognitive effects of leptin in the brain and their clinical implications. Int J Clin Pract. 2010;64:1808-12.

20. Russo VC, Metaxas S, Kobayashi K, Harris M, Werther GA. Antiapoptotic effects of leptin in human neuroblastoma cells. Endocrinology. 2004;145:4103-12.

21. Teryaeva NB. [Leptin as a neuroprotector and functional stability factor in the central neural system]. Ross Fiziol $\mathrm{Zh} \mathrm{Im} \mathrm{I} \mathrm{M}$ Sechenova. 2013;99:1138-48.

22. O'Malley D, MacDonald N, Mizielinska S, Connolly CN, Irving AJ, Harvey J. Leptin promotes rapid dynamic changes in hippocampal dendritic morphology. Mol Cell Neurosci. 2007; 35:559-72.

23. Udagawa J, Nimura $\mathbf{M}$, Otani $\mathrm{H}$. Leptin affects oligodendroglial development in the mouse embryonic cerebral cortex. Neuro Endocrinol Lett. 2006;27:177-82.

24. Procaccini C, Santopaolo M, Faicchia D, et al. Role of metabolism in neurodegenerative disorders. Metabolism. 2016;65:1376-90.

25. Lieb W, Beiser AS, Vasan RS, et al. Association of plasma leptin levels with incident Alzheimer disease and MRI measures of brain aging. JAMA. 2009;302:2565-72. 
26. Bonda DJ, Stone JG, Torres SL, et al. Dysregulation of leptin signaling in Alzheimer disease: evidence for neuronal leptin resistance. J Neurochem. 2014;128:162-72.

27. Paz-Filho GJ, Babikian T, Asarnow R, et al. Leptin replacement improves cognitive development. PLoS ONE. 2008;3:e3098.

28. Hadley KB, Ryan AS, Nelson EB, Salem N. An assessment of dietary docosahexaenoic acid requirements for brain accretion and turnover during early childhood. World Rev Nutr Diet. 2009;99:97-104.

29. Innis SM. Dietary omega 3 fatty acids and the developing brain. Brain Res. 2008;1237:35-43.

30. Joffre C, Nadjar A, Lebbadi M, Calon F, Laye S. n-3 LCPUFA improves cognition: the young, the old and the sick. Prostaglandins Leukot Essent Fat Acids. 2014;91:1-20.

31. Kuratko CN, Barrett EC, Nelson EB, Salem N Jr. The relationship of docosahexaenoic acid (DHA) with learning and behavior in healthy children: a review. Nutrients. 2013;5:2777-810.

32. Bazan NG, Molina MF, Gordon WC. Docosahexaenoic acid signalolipidomics in nutrition: significance in aging, neuroinflammation, macular degeneration, Alzheimer's, and other neurodegenerative diseases. Annu Rev Nutr. 2011;31:321-51.

33. Freund-Levi Y, Eriksdotter-Jonhagen M, Cederholm T, et al. Omega-3 fatty acid treatment in 174 patients with mild to moderate Alzheimer disease: OmegAD study: a randomized doubleblind trial. Arch Neurol. 2006;63:1402-8.

34. Schaefer EJ, Bongard V, Beiser AS, et al. Plasma phosphatidylcholine docosahexaenoic acid content and risk of dementia and Alzheimer disease: the Framingham Heart Study. Arch Neurol. 2006;63:1545-50.

35. Morris MC, Evans DA, Tangney CC, Bienias JL, Wilson RS. Fish consumption and cognitive decline with age in a large community study. Arch Neurol. 2005;62:1849-53.

36. Rapoport SI, Rao J, Igarashi M. Brain metabolism of nutritionally essential polyunsaturated fatty acids depends on both the diet and the liver. Prostaglandins Leukot Essent Fat Acids. 2007; 77:251-61.

37. Bazinet RP, Laye S. Polyunsaturated fatty acids and their metabolites in brain function and disease. Nat Rev Neurosci. 2014;15:771-85.

38. Quinn JF, Raman R, Thomas RG, et al. Docosahexaenoic acid supplementation and cognitive decline in Alzheimer disease: a randomized trial. JAMA. 2010;304:1903-11.

39. Casanas-Sanchez V, Perez JA, Fabelo N, Quinto-Alemany D, Diaz ML. Docosahexaenoic (DHA) modulates phospholipidhydroperoxide glutathione peroxidase (Gpx4) gene expression to ensure self-protection from oxidative damage in hippocampal cells. Front Physiol. 2015;6:203.

40. Calderon F, Kim HY. Docosahexaenoic acid promotes neurite growth in hippocampal neurons. $J$ Neurochem. 2004;90:979-88.

41. Moriguchi T, Harauma A, Salem N Jr. Plasticity of mouse brain docosahexaenoic acid: modulation by diet and age. Lipids. 2013;48:343-55.

42. Lei E, Vacy K, Boon WC. Fatty acids and their therapeutic potential in neurological disorders. Neurochem Int. 2016; 95:75-84.

43. Novak EM, Dyer RA, Innis SM. High dietary omega-6 fatty acids contribute to reduced docosahexaenoic acid in the developing brain and inhibit secondary neurite growth. Brain Res. 2008; 1237:136-45.

44. van Elst K, Bruining H, Birtoli B, Terreaux C, Buitelaar JK, Kas MJ. Food for thought: dietary changes in essential fatty acid ratios and the increase in autism spectrum disorders. Neurosci Biobehav Rev. 2014;45:369-78.

45. Wijendran V, Downs I, Srigley CT, et al. Dietary arachidonic acid and docosahexaenoic acid regulate liver fatty acid desaturase (FADS) alternative transcript expression in suckling piglets. Prostaglandins Leukot Essent Fat Acids. 2013;89:345-50. 